\title{
PERFIL DOS FUTUROS AUXILIARES DE ENFERMAGEM DA CIDADE DE CAMPINAS, SP, EM 1995: MOTIVOS, EXPECTATIVAS E DIFICULDADES RELACIONADAS AO CURSO*
}

\author{
Rosely Moralez de Figueiredo** \\ Maria Andreia Silva***
}

FIGUEIREDO, R.M. de; SILVA, M.A. Perfil dos futuros auxiliares de enfermagem da cidade de Campinas, SP, Brasil, em 1995: motivos, expectativas e dificuldades relacionadas ao curso. Rev.latino-am.enfermagem, Ribeirão Preto, v. 5, n. 1, p. 89-96, janeiro 1997.

O presente trabalho tem por objetivo caracterizar o perfil dos estudantes dos cursos de auxiliar de enfermagem da cidade de Campinas. As características predominantes foram: faixa etária 18 e 30 anos, maioria do sexo feminino, solteira e religião católica. A maior parte da população estudada não trabalha na área de enfermagem, sendo a principal motivação para iniciar o curso a possibilidade de ajudar outras pessoas. Como dificuldades estão o ter que voltara estudar, conciliar os horários de estudo com outras atividades e pagar o curso.

UNITERMOS: auxiliar de enfermagem, ensino médio, enfermagem, dados demográficos

\section{INTRODUÇÃO}

No Estado de São Paulo a população de profissionais de enfermagem cadastrada no COREN (Conselho Regional de Enfermagem), até a metade do ano de 1995, é de 159. 108 indivíduos, divididos em 17.494 enfermeiros, 8.731 técnicos de enfermagem, 67.294 atendentes de enfermagem e 65.589 auxiliares de enfermagem ${ }^{3}$. O auxiliar é, portanto, a segunda maior categoria na população de enfermagem atuante no mercado de trabalho sendo superada apenas pelo atendente de enfermagem. A categoria atendente de enfermagem está em processo de profissionalização, justificando, assim, a obtenção de um maior conhecimento acerca dos cursos para auxiliares de enfermagem ${ }^{1,5,6,8}$. A falta de qualidade e quantidade de profissionais de enfermagem no Brasil ${ }^{11}$ é também uma preocupação de todos aqueles que trabalham ou pesquisam nesta área, aumentando assim o interesse por estudos desta natureza.

Observa-se que a demanda pelos cursos de auxiliar de enfermagem aumentou muito nos últimos anos $\mathrm{e}$ isto pode ser evidenciado pelo número crescente de novas escolas que surgem a cada dia em todo o Estado, em especial na cidade de Campinas onde foram criados três novos cursos nos últimos cinco anos e o número de vagas amplia-se ao início de cada nova turma. Este aumento poderia ser explicado pela necessidade de regularização da situação dos atuais atendentes de enfermagem e/ou por uma forma de se obter uma profissão de nível médio com um amplo mercado de trabalho ${ }^{5}$.

Entretanto, não se observa a mesma expansão, encontrada no número de escolas, nas pesquisas sobre este profissional, suas reais necessidades de formação e do mercado de trabalho. PORFÍRIO et $\mathrm{al}^{9}$ relata a dificuldade em se encontrar material bibliográfico a respeito do ensino para auxiliares e técnicos de enfermagem. Acrescenta-se a isto o não preparo do professor, uma vez que grande parte deles não possui formação específica (licenciatura) e exerce suas atividades baseada apenas em sua experiência clínica. OLIVEIRA ${ }^{7}$ questiona também a formação do licenciado em enfermagem, que repete o modelo de poder do enfermeiro sobre o auxiliar e a posição do auxiliar de enfermagem como o responsável pelo fazer sem qualquer contribuição intelectual ao processo, acentuando assim os conflitos de classe.

$\mathrm{Na}$ cidade de Campinas existem seis escolas com o curso de auxiliar de enfermagem, sendo lançados no

\footnotetext{
* Trabalho apresentado no "1 Encontro de Professores do Ensino UNICAMP, Campinas, 22/09/95 Médio de Enfermagem: desafios de sua formação e prática".

** Professora do Curso de Auxiliar de Enfermagem do Centro Social Presidente Kennedy e pós-graduanda (doutorado) em Saúde Mental, Faculdade de Ciências Médicas, UNICAMP

*** Professora do Curso de Auxiliar de Enfermagem do Centro Social Presidente Kennedy e enfermeira do Serviço de Educação Continuada, Hospital Municipal Mano Gatti
} 
mercado de trabalho cerca de 350 novos profissionais todos os anos. Quem são nossos futuros auxiliares de enfermagem? O presente estudo objetiva:

1 - Verificar características epidemiológicas dos estudantes de auxiliar de enfermagem,

2 - Levantar motivos pelos quais eles procuram o curso, e

3 - Levantar expectativas e dificuldades relacionadas ao curso.

\section{METODOLOGIA}

O presente trabalho foi realizado em várias escolas com o objetivo de se conseguir um maior número de respostas. Como não se procurou caracterizar as escolas individualmente, os dados foram analisados como um único grupo por meio de estatística descritiva. Para tanto foi elaborado um questionário (Anexo 1), composto por doze questões de múltipla escolha e espaço para opiniões livres. Este instrumento foi testado em uma população de quinze alunos que não se encontravam dentro dos critérios de inclusão e, portanto não participaram da pesquisa.

Os questionários foram aplicados em quatro das seis escolas dos cursos de auxiliar de enfermagem da cidade de Campinas, estado de São Paulo. Estas quatro escolas foram escolhidas por serem as que se enquadravam nos critérios de inclusão, ou seja, seus alunos estavam com mais de $30 \%$ do curso concluído e concordaram em participar da pesquisa. Os dados foram coletados no período de julho a outubro de 1995.

As pesquisadoras contataram as escolas através dos coordenadores de curso e após a permissão da realização do trabalho se dirigiam para a sala de aula onde o professor distribuía o instrumento a todos os presentes. Ao final da aula os mesmos eram recolhidos pelas pesquisadoras. Todos os questionários distribuídos foram respondidos.

\section{RESULTADOS E DISCUSSÃO}

Das quatro escolas que participaram da pesquisa duas são particulares ( $A$ e $C$ ) e duas gratuitas (B e $D$ ), sendo as duas últimas mantidas por hospitais da cidade.

O total de questionários aplicados e recolhidos foi de 240. A distribuição final ficou assim estabelecida: escola A (Centro Social Presidente Kennedy): 79 alunos entrevistados, escola B (Universidade Estadual de Campinas): 88, escola C (Pontifícia Universidade Católica de Campinas): 41; e escola D (Centro Médico Campinas): 32 alunos entrevistados.

Os resultados estão apresentados em forma de tabelas seguidos de discussão dos temas abordados.
Tabela 1 - Distribuição dos estudantes dos cursos de auxiliar de enfermagem segundo faixa etária.

Campinas, 1995

\begin{tabular}{c|c|c}
\hline Idade (em anos) & $\mathbf{N}^{\mathbf{0}}$ & $\mathbf{\%}$ \\
\hline $18-30$ & 140 & 58,34 \\
$31-40$ & 66 & 27,50 \\
$41-50$ & 32 & 13,33 \\
+ de 50 & 02 & 0,83 \\
Total & 240 & 100,00 \\
\hline
\end{tabular}

$\mathrm{Na}$ faixa etária entre 18 e 30 anos encontramse $58,34 \%$ da população entrevistada, seguida de $27,50 \%$ entre 31 e 40 anos (Tabela 1). MEYER \& GASTALDO $^{6}$ PORFÍRIO et $\mathrm{al}^{9}$ encontraram dados semelhantes quanto à distribuição de idade em outros cursos de auxiliares de enfermagem nas cidades de Porto Alegre (RS) em 1987 e São Paulo (SP) em 1989, respectivamente.

Estes dados demonstram que nossa população alvo constitui-se de adultos jovens que estão no início ou iniciando sua vida produtiva.

Tabela 2 - Distribuição dos estudantes dos cursos de auxiliar de enfermagem segundo sexo. Campinas, 1995

\begin{tabular}{c|c|c|c}
\hline & Masculino & Feminino & Total \\
\hline $\mathrm{N}^{\circ}$ & 24 & 216 & 240 \\
$\%$ & 10 & 90 & 100,00 \\
\hline
\end{tabular}

Com relação ao sexo o presente estudo confirma a tradição da enfermagem, ou seja, uma profissão predominantemente feminina ${ }^{11}$. Dos alunos entrevistados $90 \%$ são do sexo feminino (Tabela 2). Estes dados repetem exatamente a mesma proporção encontrada por PORFÍRIO et al. ${ }^{9}$ na cidade de São Paulo (SP) no ano de 1989 e se aproximam dos achados de MEYER \& GASTALDO ${ }^{6}$ Ritter apud MEYER \& GASTALDO ${ }^{6}$, na cidade de Porto Alegre (RS) no ano de 1987, que afirmam serem estes cursos de predominância absoluta feminina.

Tabela 3 - Distribuição dos estudantes dos cursos de auxiliar de enfermagem segundo grau de escolaridade. Campinas, 1995

\begin{tabular}{l|c|c|c|c|c|c}
\hline & $\begin{array}{c}1^{\circ} \text { grau } \\
\text { completo }\end{array}$ & $\begin{array}{c}2^{\circ} \text { grau } \\
\text { incompleto }\end{array}$ & $\begin{array}{c}2^{\circ} \text { grau } \\
\text { completo }\end{array}$ & $\begin{array}{c}3^{\circ} \text { grau } \\
\text { incompleto }\end{array}$ & $\begin{array}{c}3^{\circ} \text { grau } \\
\text { completo }\end{array}$ & Total \\
\hline $\mathrm{N}^{\circ}$ & 82 & 56 & 80 & 16 & 06 & 240 \\
$\%$ & 34,16 & 23,34 & 33,34 & 6,66 & 2,50 & 100,00 \\
\hline
\end{tabular}

No que se refere ao grau de escolaridade constatou-se que $34,16 \%$ possuem apenas o primeiro grau completo, escolaridade esta exigida para ingresso no curso. Entretanto, 33,34\% concluíram o segundo grau e 
surpreendentemente foram encontrados 22 alunos $(9,16 \%)$ que tiveram acesso ao terceiro grau (Tabela 3$)$.

Em comparação aos dados levantados por PORFIRIO et al. ${ }^{9}$ observa-se um aumento importante do número de alunos que concluíram o segundo grau, uma vez que em seu trabalho os autores encontraram que apenas $11,04 \%$ da população estudada o tinham. Pode-se esperar, portanto, uma melhora no nível intelectual dos futuros auxiliares de enfermagem. Presume-se que melhorando a qualidade destes profissionais haverá possibilidade de uma maior participação dos mesmos nos processos decisórios e de criação, elevando o nível da assistência de enfermagem.

Tabela 4 - Distribuição dos alunos dos cursos de auxiliar de enfermagem segundo estado civil. Campinas, 1995

\begin{tabular}{l|c|c|c|c|c|c}
\hline & Casado & Solteiro & Viúvo & Divorciado & Outros & Total \\
\hline $\mathrm{N}^{\circ}$ & 98 & 106 & 04 & 19 & 13 & 240 \\
$\%$ & 40,83 & 44,17 & 1,67 & 7,92 & 5,41 & 100,00 \\
\hline
\end{tabular}

Quando ao estado civil constatou-se que quase metade da população $(44,17 \%)$ é solteira, sendo seguida pela população casada $(40,83 \%)$ (Tabela 4). Estes dados reforçam os encontrados por PORFíRIO et al $^{9}$.

Tabela 5 - Distribuição dos alunos dos cursos de auxiliar de enfermagem segundo religião. Campinas, 1995

\begin{tabular}{c|c|c|c|c|c|c}
\hline & Nenhuma & Espírita & Evangélica & Católica & Outras & Total \\
\hline $\mathrm{N}^{0}$ & 11 & 17 & 57 & 143 & 12 & 240 \\
$\%$ & 4,58 & 7,08 & 23,75 & 59,59 & 5,00 & 100,00 \\
\hline
\end{tabular}

No aspecto religião $59,59 \%$ das entrevistas assinalaram serem católicas e 23,75\% evangélicas (Tabela 5). Esta questão foi elaborada na tentativa de correlacionar um possível aumento do número de adeptos de determinadas religiões na área de enfermagem. Entretanto, aparentemente a proporção se mantém a mesma que na população geral, ou seja, com uma tendência ao aumento das religiões evangélicas que alcança quase $50 \%$ do número de assinalantes católicos ${ }^{10}$.

Tabela 6 - Distribuição dos alunos dos cursos de auxiliar de enfermagem segundo o trabalho ou não na área de enfermagem antes do início do curso. Campinas, 1995

\begin{tabular}{c|c|c|c}
\hline & Sim & Não & Total \\
\hline $\mathrm{N}^{\circ}$ & 72 & 168 & 240 \\
$\%$ & 30 & 70 & 100,00 \\
\hline
\end{tabular}

Dos alunos entrevistados $70 \%$ não trabalhavam na área de enfermagem antes de iniciarem o curso (Tabela 6). Estes dados mostram que, na população estudada, existe uma maior procura pelo curso por indivíduos que não atuam na enfermagem, ou seja, os cursos estão formando novos profissionais e não regularizando a situação dos atuais atendentes de enfermagem. Isto é uma contradição, uma vez que a maioria dos trabalhadores em enfermagem ainda é composta de atendentes ${ }^{3}$.

Estes dados diferem dos encontrados por MEYER \& GASTALDO $^{6}$ e OLIVEIRA $^{7}$, onde as populações estudadas pelas autoras eram na sua maioria atendentes de enfermagem. Diante deste fato pensamos: como e onde estão os 67.294 atendentes do Estado de São Paulo? Estarão eles sentindo necessidade de profissionalização? Será esta realidade diferente em outras cidades do Estado? Estas questões, com certeza, reforçam a necessidade de mais estudos nesta área.

Tabela 7 - Distribuição das respostas dos 72 alunos dos cursos de auxiliar de enfermagem que já trabalhavam na área, segundo a motivação que os levaram a fazer o curso. Campinas, 1995

\begin{tabular}{l|c|c}
\hline Motivação & $\begin{array}{c}\mathrm{N}^{\circ} \text { de } \\
\text { resposta }\end{array}$ & $\%$ \\
\hline a. Necessidade de regularizar a profissão & 33 & 33 \\
b. Exigência do local de trabalho & 08 & 08 \\
c. Necessidade de melhorar o conhecimento & 55 & 55 \\
d. Outros & 04 & 04 \\
Total & 100 & 100 \\
\hline
\end{tabular}

Os 72 alunos que já trabalhavam na enfermagem antes de iniciarem o curso de auxiliar alegaram que começaram o curso para regularizarem suas situações (33\% das respostas) e melhorarem seus conhecimentos (55\%) (Tabela 7). Estes dados diferem dos encontrados por RITTER apud MEYER \& GASTALDO ${ }^{6}$ onde $32,67 \%$ dos entrevistados apontou querer regularizar a profissão, 22,67\% melhorar o salário e 19,37\% melhorar o conhecimento.

Observa-se também que apenas 11\%, dos 72 alunos que já trabalhavam na enfermagem, ou seja, oito alunos, procuraram o curso por exigência das instituições onde trabalham, demonstrando a falta de preocupação por parte dos empregadores com a profissionalização do atendente. Isto está, portanto, em desacordo com os objetivos iniciais do aumento do número de escolas de auxiliar de enfermagem, que segundo MEYER \& GASTALDO ${ }^{6}$, visavam profissionalizar os práticos de enfermagem atuantes no mercado de trabalho. Percebe-se também que a Lei 7.498/86 que estabelecia um período 
de dez anos para regularizara situação destes profissionais e que somente em dezembro de 1994 foi revogada pela Lei $8.967 / 94^{2}$, não pressionou significativamente as instituições empregadoras no sentido de facilitarem e incentivarem a qualificação de seus atendentes.

Tabela 8 - Distribuição das respostas dos 168 alunos dos cursos de auxiliar de enfermagem que não trabalhavam na área, segundo a motivação que os levaram a fazer o curso. Campinas, 1995

\begin{tabular}{l|c|c}
\hline Motivação & $\begin{array}{c}\mathrm{N}^{0} \text { de } \\
\text { resposta }\end{array}$ & $\%$ \\
\hline a. Interesse pela área & 144 & 58,30 \\
b. Ter uma profissão & 61 & 24,70 \\
c. Maior chance de arrumar emprego & 24 & 9,72 \\
d. A única opção que surgiu & 02 & 0,80 \\
e.Outros & 16 & 6,48 \\
Total & 247 & 100 \\
\hline
\end{tabular}

Obs: O aluno poderia optar por mais de uma resposta

Quando foi perguntado porque as pessoas que não trabalhavam na área resolveram fazer o curso 5 $8,30 \%$ das respostas afirmaram por ter interesse pela área, contra $24,70 \%$ que desejavam uma profissão e $9,72 \%$ que visavam a possibilidade de emprego (Tabela 8). Considerando que os alunos poderiam marcar mais de uma resposta, percebe-se que o interesse pela área ainda é apontado como propulsor para iniciar o curso e não a possibilidade de se ter uma profissão ou conseguir um emprego. Pode-se ver ainda que 16 alunos $(6,48 \%)$ alegaram outros motivos para iniciarem o curso como: mudar de vida, ajudar outras pessoas, suporte para iniciar a faculdade e falta de opção, entre outros.

Tabela 9 - Distribuição das respostas dos alunos dos cursos de auxiliar de enfermagem segundo suas expectativas em relação ao curso. Campinas, 1995

\begin{tabular}{l|c|c}
\hline Expectativas & $\begin{array}{c}\mathrm{N}^{\circ} \text { de } \\
\text { resposta }\end{array}$ & $\%$ \\
\hline a. Realizar o sonho de ser aux. de enfermagem & 72 & 19,84 \\
b. Conseguir um bom emprego & 27 & 7,44 \\
c. Ter uma profissão & 82 & 22,58 \\
d. Poder ajudar outras pessoas & 166 & 45,74 \\
e.Outros & 16 & 4,40 \\
Total & 363 & 100 \\
\hline
\end{tabular}

Obs: O aluno poderia optar por mais de uma resposta

No que se refere às perspectivas que os aluno têm com o curso $45,74 \%$ das respostas se relaciona com a possibilidade de ajudar outras pessoas, $22,58 \%$ com a de ter urna profissão e $19,84 \%$ com a de realizar o sonho de ser auxiliar de enfermagem (Tabela 9). Observa-se que quase a metade das respostas ainda se refere ao curso de auxiliar de enfermagem como urna forma de ajudar as pessoas.

Segundo DANIEL ${ }^{4}$ a tarefa primordial da enfermagem é ajudar outras pessoas, utilizando para isto, de modo calculado, o sentir, o pensar e o agir no relacionamento interpessoal, superando a forma casual, impensada, automática e impessoal de lidar com o ser humano ${ }^{4}$. Partindo do princípio de que esta é a perspectiva principal do aluno que procura o curso de auxiliar de enfermagem, será que estamos conseguindo aproveitar esta pré disposição do aluno? A relação de ajuda é discutida e encarada como foco principal desta profissão? Será que às portas do ano 2000 ainda não conseguimos encontrar uma postura equilibrada entre a competência técnica e a relação de ajuda desvinculando a enfermagem da caridade? A direção em que a profissão caminha está também relacionada à visão dos profissionais que formamos.

Tabela 10 - Distribuição dos alunos dos cursos de auxiliar de enfermagem segundo o atendimento das expectativas em relação ao curso. Campina 1995

\begin{tabular}{l|c|c}
\hline Atendimento de expectativas & $\mathrm{N}^{\circ}$ & $\%$ \\
\hline a. Sim & 157 & 65,42 \\
b. Não & 04 & 1,66 \\
c. Em parte & 36 & 15,00 \\
d. Supera & 43 & 17,92 \\
e.Outros & 00 & 0,00 \\
Total & 240 & 100 \\
\hline
\end{tabular}

Quanto as expectativas dos alunos entrevistados $83,34 \%$ consideram que o curso está atendendo suas expectativas e apenas 1,66\% referem que não (Tabela 10). 
Tabela 11 - Distribuição dos alunos dos cursos de auxiliar de enfermagem segundo as dificuldades encontradas no curso. Campinas, 1995

\begin{tabular}{l|c|c}
\hline Dificuldades encontradas & $\mathrm{N}^{\circ}$ & $\%$ \\
\hline a. Voltar a estudar & 43 & 17,91 \\
b. Acompanhar parte teórico & 44 & 18,33 \\
c. Executar técnicas de enfermagem & 27 & 11,25 \\
d. Relacionar-se como pacientes e & 10 & 4,17 \\
funcionários do hospital & & \\
e. Relacionamento com os professores & 10 & 4,17 \\
f. Outros & 66 & 27,50 \\
g. Sem respostas & 40 & 16,67 \\
Total & 240 & 100 \\
\hline
\end{tabular}

Obs: Alguns alunos não assimilaram dificuldades

$\mathrm{Na}$ Tabela 11, quanto às dificuldades encontradas pelos alunos, $21,50 \%$ refere-se ao fato de terem que retornar a estudar, $22 \%$ ao de acompanhar a parte teórica e $13,50 \%$ ao de executar as técnicas de enfermagem. Entretanto, 60 alunos (33\%) alegam outros motivos sendo a conciliação do tempo de estudo com outras atividades (emprego, filhos e casa, entre outras), o pagamento do curso (nas escolas particulares) e o meio de transporte, como sendo as maiores dificuldades. Estes achados podem ser explicados por se tratar de uma população predominantemente feminina, dependente de seus próprios salários, ou seja, acabam exercendo uma tripla jornada, como aluna, mãe, dona de casa e trabalhadora ${ }^{6,7}$.

Esta sobrecarga de funções pode ainda ser o principal motivo pelo qual tão poucos atendentes conseguem ter acesso à sua profissionalização.

\section{CONCLUSÕES}

Os dados obtidos neste estudo indicam que nas escolas pesquisadas, na cidade de Campinas (SP), a população que procura os cursos de auxiliar de enfermagem é formada por adultos jovens (entre $18 \mathrm{e}$ 30 anos), predominantemente feminina, sendo a metade composta por solteiros, católicos e 1/3 com segundo grau completo (escolaridade acima da mínima exigida no curso, com 9,16\% inclusive tendo acesso ao terceiro grau). A maioria desta população não trabalhava na área de enfermagem antes do início do curso, mostrando que a profissionalização do atendente, neste estudo, não está ocorrendo da forma como se esperava. A principal motivação relatada é a possibilidade de ajudar outras pessoas e apontam como dificuldades: conciliar atividades domésticas, trabalho e estudo e o pagamento do curso.

Agradecimentos: As autoras agradecem a leitura crítica e sugestões apresentadas por dois revisores anônimos a uma versão preliminar do artigo.

\section{PROFILE OF FUTURE AUXILIARY NURSES IN THE CITY OF CAMPINAS, SP, IN 1995: REASONS, EXPECTATION AND DIFFICULTIES RELATED TO THE COURSE}

The present study aims to characterize the profile of people studying in auxiliary nurse courses at the city of Campinas. The predominantly characteristics were: ages between 18 and 30 years old, majority female, single and catholic. The major part of the studied population does not work in nursing area, being the mainly motivation to begin the course the possibility of helping other people. Their difficulties were returning to school, conciliating the study schedule with other activities and paving the course.

\section{PERFIL DE LOS FUTUROS AUXILIARES DE ENFERMERÍA EN LA CIUDAD DE CAMPINAS, SP, EN 1995: MOTIVOS, EXPECTATIVAS Y OBSTÁCULOS RELACIONADOS AL CURSO}

El presente trabajo tiene como objetivo caracterizar el perfil de los estudiantes de los cursos de auxiliar de enfermería en la ciudad de Campinas. Las características que predominaron fueron: una faja etaria entre 18 y 30 años, mayoría del sexo femenino, soltera y de religión católica. La mayor parte de la población estudiada no trabaja en el área de enfermería, siendo el principal motivación para iniciar el curso la posibilidad de ayudar otros. Como obstáculos se encontraron: el hecho de tener que volver a estudiar, conciliar los horarios de estudio con otras actividades y, finalmente pagar el curso. 


\section{REFERÊNCIAS BIBLIOGRÁFICAS}

01. BASTOS, V.L.S. Avaliação de um programa de capacitação de recursos humanos em enfermagem para a zona rural. Rev.Bras.Enfermagem, v. 34, p. 260-268. 1981.

02. BOLETIM INFORMATIVO COREN-SP, n. 1, 1995.

03. BOLETIM INFORMATIVO COREN-SP, n. 2, 1995.

04. DANIEL, L.F. Atitudes interpessoais em enfermagem. São Paulo: EPU, 1983. 176 p.

05. DOURADO, H.G. Oportunidade de educação para o pessoal numeroso de enfermagem: Exames Supletivos (editorial). Rev. Bras. Enfermagem, v. 30, p. 71-72, 1977.

06. MEYER, D.E.; GASTALDO, D. Qualificação do auxiliar de enfermagem: um conflito entre formação e a realidade profissional. Ci. e Cult., v. 41, p. 171-176. 1989.
07. OLIVEIRA, M.W. A relação entre licenciatura em enfermagem e a problemática do exercício profissional em equipe. IApresentado no I Encontro de Professores do Ensino Médio em Enfermagem: Desafios ele sua Formação e Prática. Faculdade. de Educação, UNICAMP, Campinas, 1995.

08. OGUISSO, T. Considerações sobre a legislação do ensino e do exercício e do técnico de enfermagem e do auxiliar de enfermagem. Rev. Bras.Enfermagem., v. 30, p. 168-174. 1977.

09. PORFíRIO, R.M. et al. Perfil sócio-econômicocultural do estudante de auxiliar de enfermagem de São Paulo - SP. Rev.Bras.Enfermagem, v. 45, p. 290-301, 1992.

10. SÁ, X. Deus de resultados. Folha de São Paulo, 5/11/1995, p. 1-16

11. VIETTA, E.P., et al. Tomada de depoimento pessoal de enfermeiras hospitalares da década de 50: subsídios para a compreensão da enfermagem atual. Rev.latino-am.enfermagem, v. 3, p. 1935. 1995. 


\section{Anexo 1 - Questionário aplicado a estudantes de cursos de auxiliar de enfermagem da cidade de Campinas}

1) Idade:
A ( ) 18-30 anos
C ( ) 41-50 anos
B ( ) 31-40 anos
$\mathrm{D}(\mathrm{)}+\mathrm{de} 50$ anos

2) Sexo:

A ( ) masculino $\quad$ C ( ) feminino

3) Escolaridade
A ( ) $1^{\circ}$ grau completo
C ( ) $3^{\circ}$ grau incompleto
$\mathrm{B}(\mathrm{C}) 2^{\circ}$ grau incompleto
$\mathrm{D}\left(\mathrm{)} 3^{\circ}\right.$ grau completo

C ( ) $2^{\circ}$ grau completo

4) Estado civil
A ( ) casado
C ( ) $3^{\circ}$ grau incompleto
E ( ) outros
$\mathrm{B}(\mathrm{)})$ solteiro
D ( ) $3^{\circ}$ grau completo
Qual?

5) Você tem alguma religião? Qual?
A ( ) nenhuma
B ( ) espírita
C ( ) evangélica
D ( ) católica
E ( ) outra. Qual?

6) Você já trabalhava na área de enfermagem antes de começar este curso?
A ( ) sim
C ( ) não

7) Caso você tenha respondido sim na pergunta anterior, responda: Por que você resolveu fazer este curso?

A ( ) necessidade de regularizar a profissão

$B$ ( ) exigência do local em que trabalho

C ( ) necessidade de melhorar o conhecimento

D ( ) Outros. Quais?

8) Caso você tenha respondido não na questão 6, responda: Por que você resolveu fazer este curso?

A ( ) interesse pela área

B ( ) ter uma profissão

C ( ) maior chance de arrumar emprego

D ( ) a única opção que surgiu

E ( ) outros. Qual?

9) O que você espera este curso?

$A($ ) realizar meu sonho de ser auxiliar de enfermagem

$B($ ) conseguir um bom emprego

C ( ) ter uma profissão

D ( ) poder ajudar outras pessoas

E ( ) outros. Qual?

10) O curso está atendendo suas expectativas?

$A($ ) sim

B ( ) não

C ( ) em parte

D ( ) está superando minhas expectativas

E ( ) outros. Qual? 
11) Quais as maiores dificuldades encontradas no curso?

A ( ) voltar a estudar

B ( ) acompanhar a parte teórica

C ( ) executar as técnicas de enfermagem

D ( ) relacionar-se com os pacientes e funcionários do hospital

$\mathrm{E}$ ( ) relacionamento com os professores

F ( ) outros. Qual? 